15

\title{
Двухмембранная система ввода пробы в масс-спектрометр для исследования выдыхаемого воздуха
}

\author{
(C) В. Коган, ${ }^{1}$ А.С. Антонов, ${ }^{1}$ Ю.В. Чичагов, ${ }^{1}$ О.С. Викторова-Леклерк, ${ }^{1}$ И.В. Викторов, ${ }^{1}$ А.В. Козленок ${ }^{2}$ \\ ${ }^{1}$ Физико-технический институт им. А.Ф.Иофффе РАН, \\ 194021 Санкт-Петербург, Россия \\ 2 Федеральный медицинский исследовательский центр им. Алмазова, \\ 197341 Санкт-Петербург, Россия \\ e-mail: Viktor.Kogan@mail.ioffe.ru, vtkogan@hotmail.com
}

(Поступило в Редакцию 1 июня 2017 г. В окончательной редакции 19 фревраля 2018 г.)

Описана двухмембранная система ввода пробы, позволяющая проводить масс-спектрометрическое определение летучих органических соединений в воздухе, обеспечивая при этом пределы их обнаружения от долей миллиардной части состава пробы (долей $\mathrm{ppb}$ ) в режиме реального времени с задержкой отклика от единиц до десятков секунд. Она включает два мембранных интерфейса, разделенных объемом, давление газа в котором поддерживается через канал с регулируемым сечением откачки. Представлены результаты численного моделирования и экспериментальной проверки системы. Описана методика выбора и расчета параметров системы. Обсуждена возможность применения рассмотренной системы для диагностики заболеваний по следовым количествам биомаркеров в выдыхаемом воздухе.

DOI: $10.21883 /$ JTF.2018.10.46507.2372

\section{Введение}

Достижение низких пределов обнаружения (от долей $\mathrm{ppb}$ ) летучих органических соединений в воздушных пробах при изучении динамики процессов с характерными временами секунды и менее, например, диагностика заболеваний по составу выдыхаемого воздуха, наилучшим образом осуществляется инструментами Proton Transfer Reaction Mass-Spectrometry (PTR-MS) [1] и Selected Ion Flow Tube Mass-Spectrometry (SIFT-MS) [2]. Линейки таких инструментов включают приборы с разными возможностями и позволяют достигать пределов определения на уровне ppt [3-6], но все они отличаются сложностью процедуры измерения и дороговизной. В то же время по своим возможностям к ним приближается более простой метод масс-спектрометрии с использованием мембранных интерфейсов (Membrane Introduction Mass Spectrometry, MIMS) [7,8]. За счет избирательной проницаемости используемых мембран MIMS позволяет добиваться высокой чувствительности для значительной группы целевых соединений. В процессе введения пробы в прибор происходит обогащение воздушной пробы целевыми соединениями до тысяч раз [9]. Тем не менее применение одиночного мембранного интерфейса часто оказывается недостаточным для достижения требуемых характеристик инструмента. Так, в медицине при проведении ранней диагностики заболеваний по составу выдыхаемого воздуха, предел определения биомаркеров должен находиться на уровне долей $p p b$.

В работах [10-12] показана принципиальная возможность применения многомембранных систем ввода для увеличения эффекта относительного обогащения пробы целевыми соединениями в процессе подачи ее в массспектрометр. Однако в этих работах не определены требования к параметрам многомембранных систем и к режиму ввода пробы, которые позволили бы перейти от относительного обогащения пробы к понижению предела обнаружения целевых соединений за счет введения дополнительной мембраны в систему ввода. Кроме того, в этих работах не рассматриваются вопросы влияния параметров системы ввода на задержку отклика прибора после изменения состава пробы, что важно при изучении динамики изучаемых процессов. В настоящей работе предпринята попытка обосновать выбор параметров, обеспечивающих максимальное обогащение и быстродействие двухмембранной системы ввода пробы левеллиновского типа [13] для большой группы целевых соединений.

\section{1. Приборы и материалы}

В работе использован портативный масс-спектрометр с двойной фокусировкой, описанный в [14]. В качестве мембранного материала в работе использовались мембраны: SSP-M100, Specialty Silicone Products Inc., Ballston Spa, NY толщиной $100 \mu \mathrm{m}$ и мембраны фирмы DOW Corning толщиной $59 \mu \mathrm{m}$.

\section{2. Поток летучих соединений через двухмембранную систему}

Оригинальная система ввода пробы представлена на рис. 1. Рассмотрение ее работы в квазистатическом режиме, обеспечивающем максимальное относительное обогащение пробы соединением $j$, для которого $\sigma_{j}=\sigma_{j \max }$, целесообразно рассматривать раздельно для основного компонента воздушной пробы - азота, принимая упрощение $\sigma_{\alpha}=\sigma_{\text {Nitrogen }}$, и целевого соединения $j$ 

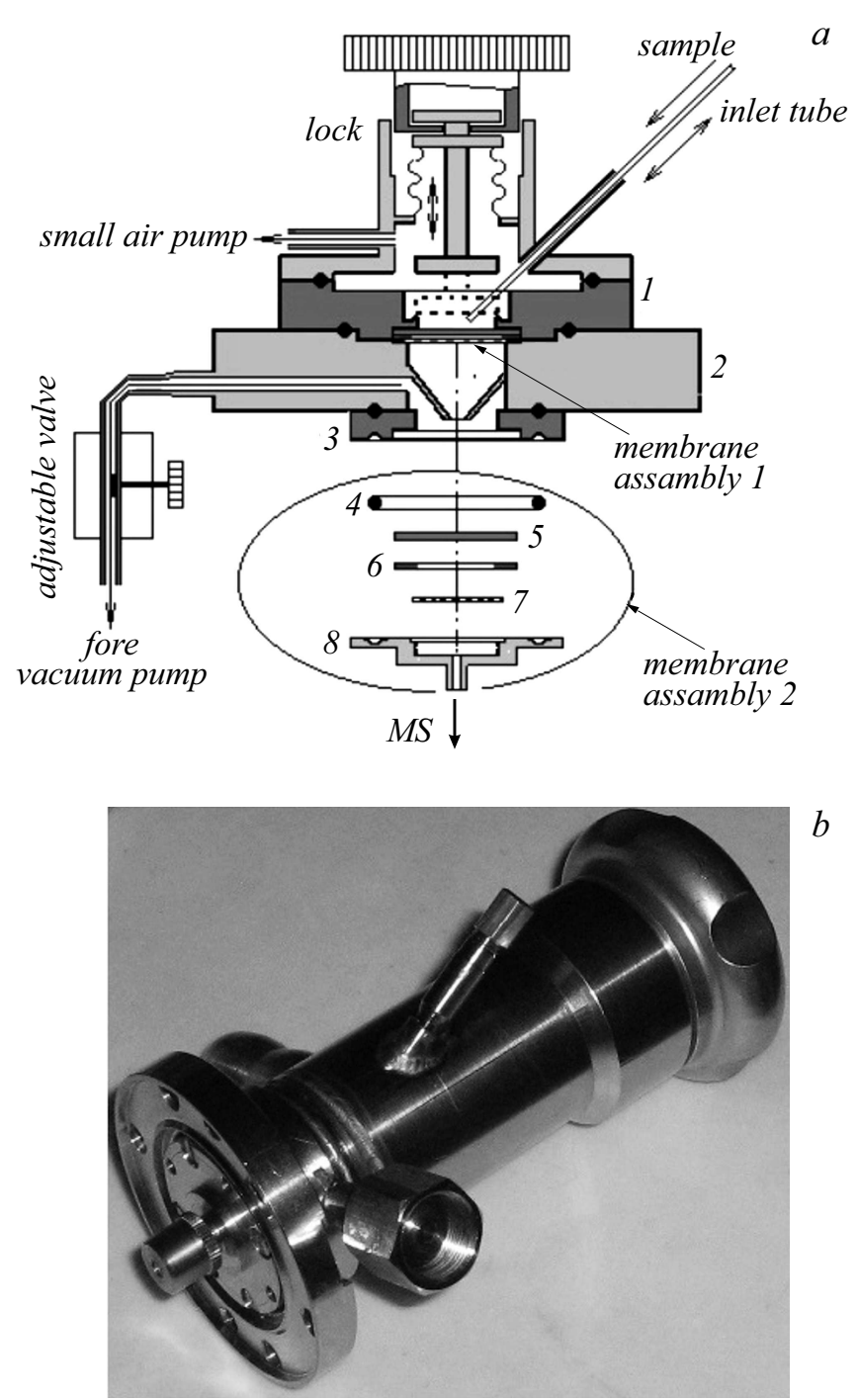

Рис. 1. Двухмембранная система ввода: $a-$ схема: $1-$ затвор, 2 - корпус системы ввода, 3 - сопрягающее кольцо, 4 - уплотнение из витона, 5 - мембрана, $6-$ силикиновое уплотнение, 7 - сетка для поддержания мембраны, $8-$ фиксирующий фланец; $b-$ внешний вид.

со следовой концентрацией. Увеличение эффекта обогащения за счет второго мембранного интерфейса определяется выбором величины отношения давлений основного компонента в исходной воздушной смеси $\left(P_{\mathrm{atm}}\right)$ и за первой мембраной $\left(P_{V}\right)$. Величина относительного обогащения пробы приближается к максимальному значению в случае выполнения условия

$$
P_{V} / P_{\mathrm{atm}} \Rightarrow \sigma_{a}^{m} / \sigma_{j \max }^{m}
$$

где $\sigma_{a}^{m}$ - величина проницаемости первой мембраны для основных компонентов воздуха, $\sigma_{j \max }^{m}-$ величина проницаемости первой мембраны для целевого соединения, обладающего наибольшей проникающей способностью через материал мембраны. Нарушение этого условия приводит к снижению эффекта обогащения пробы соединением с $\sigma_{j \max }^{m}$ из-за ограничения величины градиента его парциального давления в первой мембране.

Чтобы поддерживать выбранное значение $P_{V} / P_{\mathrm{atm}}(1)$, необходимо задать отношение проводимостей мембран $T_{a}^{m}\left(\sigma_{a}\right)$ и канала вакуумной откачки из объема между мембранами $T^{\text {branch }}$ выражением:

$$
T_{a}^{m} / T^{\text {branch }} \approx P_{V} / P_{\text {atm }}
$$

В то же время проводимости мембранных интерфейсов $T_{j}^{m}\left(\sigma_{\max }\right)$ для летучих соединений, обладающих максимальной проникающей способностью, должны быть сопоставимы с проводимостью канала вакуумной откачки $T^{\text {branch }}$, что обеспечивает попадание значительной части этих соединений в масс-спектрометр.

Эквивалентные схемы раздельного прохождения основного компонента воздуха и целевого компонента сквозь мембранную систему ввода показаны на рис. 2.

В предложенных схемах парциальные давления $P$ веществ в пробе аналогичны потенциалам $U$ в электрических схемах, сопротивления $R$ соответствуют обратным значениям проводимостей мембранных интерфейсов $T^{m}(\sigma)$ и проводимости канала вакуумной откачки $T^{\text {branch }}$ из объема $V$, а токи $I$ соответствуют потокам компонентов в системе.

Опираясь на эквивалентные схемы, выбираем оптимальные параметры, обеспечивающие эффект относительного обогащения потока за второй мембраной целевым соединением с проникающей способностью, близкой к максимальному $\sim(1 / 3)\left(\sigma_{j \max }^{m} \sigma_{a}^{m}\right)^{2}$. В то же время для целевых соединений $j$ с меньшей величиной проникающей способности $\sigma_{j}<\sigma_{j \max }$ величина относительного обогащения $E_{d, j}$ приближается к значению

$$
\left(\sigma_{j \max }^{m} / \sigma_{a}^{m}\right)^{2}
$$

Изменение величины потока целевых соединений в вакуумную камеру масс-спектрометра от времени через систему из 2-х мембран после внезапного изменения их содержания в пробе можно определить с помощью численного расчета.

$$
\begin{aligned}
& \text { j-target compound } \quad \sigma_{j}^{m} / \sigma_{a}^{m}>>1 \quad \text { a-air (nitrogen) }
\end{aligned}
$$

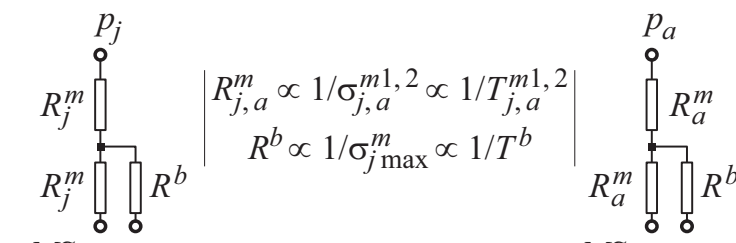

$$
\begin{aligned}
& M S \downarrow \\
& M S \downarrow \\
& I_{j} \propto p_{j} \sigma_{j \max }^{m} / 3 \\
& I_{a} \propto p_{a}\left(\sigma_{a}^{m} / \sigma_{j \max }^{m}\right) / \sigma_{a}^{m} \\
& I_{j} / I_{a} \propto\left(p_{j} / p_{a}\right)\left(\sigma_{j \max }^{m} / \sigma_{a}^{m}\right)^{2 / 3}
\end{aligned}
$$

Рис. 2. Эквивалентные схемы, для описания работы системы ввода. $j-$ целевое соединение, $a-$ воздух (компонента, преобладающая в пробе воздуха - азот), 1 - первая мембрана, 2 - вторая мембрана; $b-$ канал вакуумной откачки из межмембранного объема $V$. 


\section{Численное моделирование}

Рассмотрим процесс прохождения летучих соединений через систему ввода, состоящую из 2-х мембран, во времени. Для этого, как в работе [11], каждый из мембранных интерфейсов мы мысленно разбиваем на $n$ слоев толщиной $h$. Концентрацию газа внутри каждого слоя считаем однородной из-за малой его толщины. Непрерывное распределение концентрации компонентов по направлению их распространения заменяем дискретным набором значений матрицы $c_{i, j}$, состоящей из $2 n$ элементов, где первый индекс $i$ соответствует числу мембран, а второй $j-$ количеству слоев в них. Такие значения приписываются индексам только в данном разделе. Временной интервал нестационарного процесса диффузии от момента быстрого нарастания давления перед первой мембраной до установления стационарного процесса в ней делится на временные интервалы или шаги $\Delta t$. Если на шаге $k$ концентрация в каждом слое известна, то плотность потока компонентов между слоями может быть рассчитана из уравнения

$$
J_{i, j}^{(k)}=D \frac{c_{i, j}^{(k)}-c_{i, j+1}^{(k)}}{h} ; \quad i=1,2 ; j=1 \ldots n-1,
$$

которое является конечно-разностным аналогом 1-го уравнения Фика. Где $J_{i, j}^{(k)}-$ плотность потока на шаге $k$ из слоя $j$ в слой $j+1$. Изменение концентрации
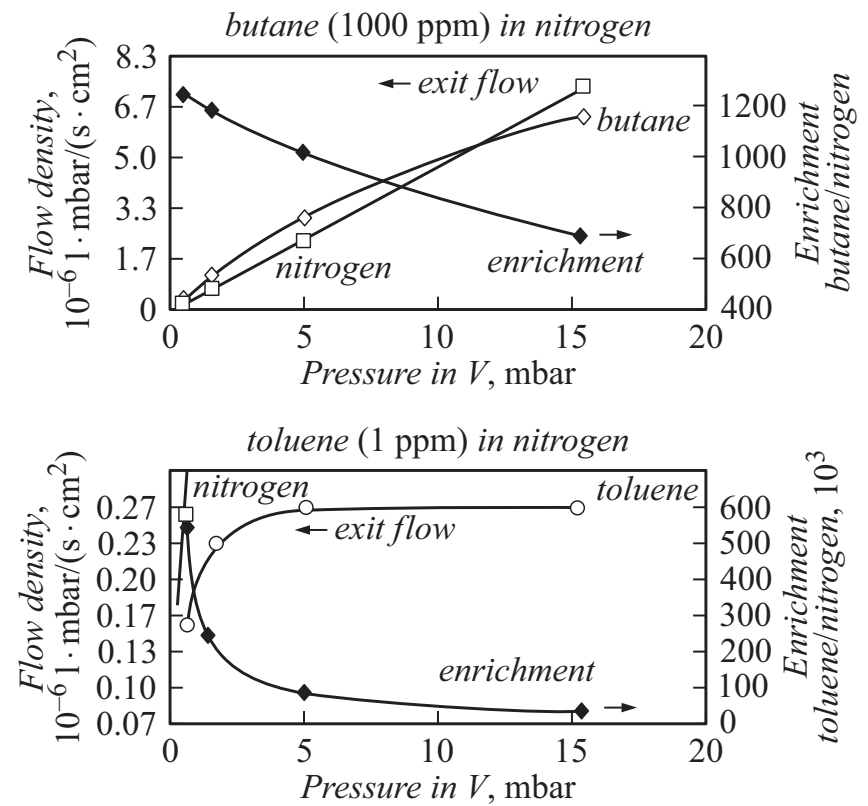

Рис. 3. Потоки плотностей азота, бутана и толуола за второй мембраной при их исходной концентрации соответственно $C_{0, N}=78 \%, C_{0 \text {, but }}=1000$ ppm, $C_{0, \text { tol }}=1$ ppm; при величинах относительной проницаемости мембран $\sigma_{\text {but }}^{m} / \sigma_{N}^{m} \sim 36$, $\sigma_{\text {tol }}^{m} / \sigma_{N}^{m} \sim 1000$ [9] и факторы обогащения пробы летучими соединениями благодаря системе ввода в зависимости от давления в межмембранном объеме $V$. Приведены результаты для мембран толщиной $30 \mu \mathrm{m}$. При использовании мембран произвольных толщин $l_{d}$ следует величчину потока плотности домножить на отношение $l_{30} / l_{d}$. компонентов внутри каждого слоя со временем рассчитывается из уравнения

$$
c_{i, j}^{(k+1)}+\Delta t \frac{J_{i, j-1}^{(k)}-J_{i, j}^{(k)}}{h} ; \quad i=1,2 ; j=1 \ldots n-1
$$

которое является конечно-разностным аналогом 2-го уравнения Фика. На границе мембраны концентрации компонентов испытывают скачок:

$$
c_{\text {in }}=\mu c_{\text {out }},
$$

величина которого определяется коэффициентом распределения $(\mu)$.

В соответствии с условием (6) концентрация внутренних слоев мембраны определяется соотношениями

$$
c_{1,1}^{(k)}=\mu \cdot C_{0}, \quad c_{1, n}^{(k)}=c_{2,1}^{(k)}=\mu \cdot C_{1}^{(k)}, \quad c_{2, n}^{(k)}=\mu \cdot C_{2},
$$

где $C_{0}=\mathrm{const}$ - концентрация газа в исходном образце $(t=0), C_{2}=0-$ концентрация газа в вакуумной камере масс-спектрометра, $C_{1}^{(k)}-$ концентрация газа в объеме между мембранами в момент времени $t=k \Delta t$, рассчитывается для каждого временного шага. Для этих целей вводится эффективный объем $V^{\prime}$, который учитывает объем между мембранами $V$ и емкость пограничных слоев соседних мембран.

В отличие от системы ввода, описанной в работе [11], в рассматриваемой системе для поддержания эффекта обогащения пробы целевыми соединениями из объема между мембранами осуществляется откачка через канал, проводимость которого определяется режимом перетекания газовой смеси. Учитывая требования к давлению в объеме $V$ в установившемся режиме и характер изменения этого давления из-за переходных процессов в мембране, можно ограничиться рассмотрением лишь ламинарного характера потока, $T^{\text {branch }} \propto P_{v}$.

Обозначим потоки, а) - втекающий в объем между мембранами $J_{1, n-1}$, b) - вытекающий из него через вторую мембрану $J_{2,1}$, и с) - через канал откачки $J_{\text {branch }}$. Тогда изменение концентрации в объеме $V$ определяется из уравнения

$$
C^{(k+1)}=C^{(k)}+\Delta t \cdot S \frac{J_{1, n-1}^{(k)}-J_{2,1}^{(k)}-J_{\text {branch }}^{(k)}}{V^{\prime}}
$$

где $V^{\prime}=V+2 S h \mu$.

Используя уравнения (4)-(8) и начальные условия $c_{1,1}^{(0)}=\mu C_{0}, \quad c_{i, j}^{(0)}=0 \quad\left(c_{1,1}^{(0)} \notin c_{i, j}^{(0)}\right)$, можно определить плотность потока компонента на выходе системы ввода пробы для любого момента времени:

$$
I_{\text {exit }}(k \Delta t)=J_{2, n-1}^{(k)} .
$$

Предложенный алгоритм позволяет рассчитать зависимости потоков компонентов в масс-спектрометр от времени после изменения их концентрации в образце. 
Таблица 1. Характерные пики масс-спектров некоторых биомаркеров заболеваний

\begin{tabular}{|c|c|c|c|c|c|c|c|}
\hline \multirow{2}{*}{ Биомаркер } & \multirow{2}{*}{$\left(\sigma_{j}^{m} / \sigma_{N}^{m}\right)$} & \multicolumn{6}{|c|}{ Болезнь } \\
\hline & & ${ }^{1} \mathrm{LC}$ & ${ }^{1} \mathrm{BC}^{3} \mathrm{LFD}$ & ${ }^{4} \mathrm{PTB}$ & ${ }^{5} \mathrm{LP}$ & ${ }^{6} \mathrm{~A}$ & \\
\hline 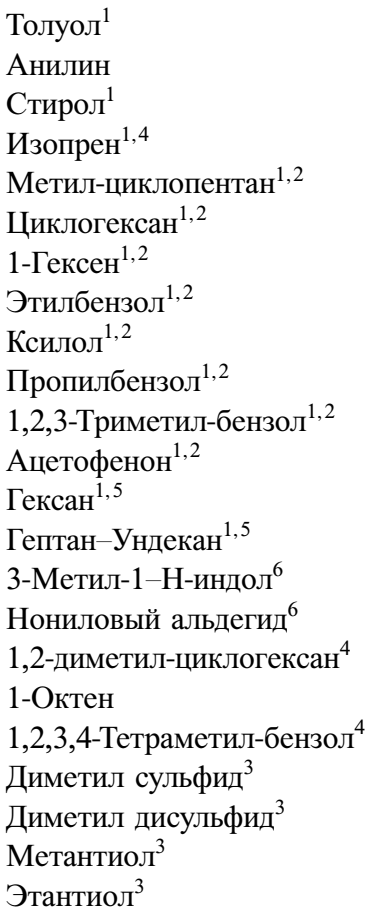 & $\begin{aligned}( & \sim 1000) \\
( & \sim 1000) \\
( & \sim 1000) \\
( & \sim 500) \\
( & \sim 800) \\
( & \sim 300) \\
( & \sim 600) \\
( & \sim 1500) \\
( & \sim 1000) \\
( & \sim 1500) \\
( & \sim 500) \\
& (?) \\
& \sim 300 \\
& \sim 800 \\
& \sim 1000 \\
& (?) \\
& \sim 500 \\
& (?) \\
& \sim 500 \\
& \sim 500 \\
& \sim 500 \\
& \sim 500 \\
& \sim 500\end{aligned}$ & $\begin{array}{c}92 \\
93 \\
104 \\
67 ; 68 \\
84 \\
84 \\
84 \\
106 \\
106 \\
120 \\
105 ; 120 \\
105 ; 120 \\
86 \\
85 \\
\\
\\
112 \\
119 ; 134\end{array}$ & $\begin{array}{c}84 \\
84 \\
84 \\
106 \\
106 \\
120 \\
105 ; 120 \\
105 ; 120\end{array}$ & $\begin{array}{c}47 ; 65 \\
94 \\
47 \\
47 ; 62\end{array}$ & $\begin{array}{c}114 ; 124 \\
97 ; 112\end{array}$ & $\begin{array}{l}86 \\
85\end{array}$ & $130 ; 131$ \\
\hline
\end{tabular}

Примечание . ${ }^{1} \mathrm{LC}-$ рак легких, ${ }^{2} \mathrm{BC}-$ рак молочной железы, ${ }^{3} \mathrm{LFD}-$ дисфункция печени, ${ }^{4} \mathrm{PTB}-$ туберкулез, ${ }^{5} \mathrm{LP}-$ окислительная деградация липидов (последствие ралиоактивного облучения), ${ }^{6} \mathrm{~A}-$ аминурия.

Таблица 2. Относительные проницаемости мембран для тестовых соединений и факторы обогащения пробы этими соединениями

\begin{tabular}{|c|c|c|c|c|}
\hline \multirow{2}{*}{ Тип системы ввода } & \multirow{2}{*}{ Тип мембраны } & \multirow{2}{*}{$\begin{array}{c}\text { Относительная проницаемость } \\
\text { мембраны по сравнению с азотом }\end{array}$} & \multicolumn{2}{|c|}{ Фактор обогащения относительно азота } \\
\hline & & & расчет & эксперимент \\
\hline \multirow{2}{*}{$\begin{array}{l}\text { Система ввода } \\
\text { с одной мембраной }\end{array}$} & $\begin{array}{l}\text { SSP-M100 } \\
\text { or SSP-M823 [18] }\end{array}$ & $\begin{array}{l}\text { Кислород } 2[18] \\
\text { Этан } \sim 8.5[18] \\
\text { Бутан } \sim 30[18] \\
\text { Толуол } \sim 30.5[18] \\
\end{array}$ & & $\begin{array}{l}\sim 2[19] \\
\sim 10[19] \\
\sim 30[19] \\
\sim 30.5[14]\end{array}$ \\
\hline & Dow Corning & $\begin{array}{l}\text { Кислород } 2[9] \\
\text { Этан } \sim 11.8[9] \\
\text { Бутан } \sim 36[9] \\
\text { Толуол } \sim 964[9]\end{array}$ & & $\begin{array}{l}\sim 2[9] \\
\sim 11.8[9] \\
\sim 36[9] \\
\sim 964[9]\end{array}$ \\
\hline \multirow{2}{*}{$\begin{array}{l}\text { Система ввода } \\
\text { с двумя мембранами }\end{array}$} & $\begin{array}{l}\text { SSP-M100 } \\
\text { or SSP-M823 }\end{array}$ & $\begin{array}{l}\text { Кислород } 2[18] \\
\text { Этан } \sim 8.5[18] \\
\text { Бутан } \sim 30[18] \\
\text { Толуол } \sim 30.5[18]\end{array}$ & $\begin{array}{l}\text { Кислород } 4 \\
\text { Этан up to } 100 \\
\text { Бутан up to } 800 \\
\text { Толуол up to } 850\end{array}$ & $\begin{array}{l}\sim 4^{*} \\
\sim 117^{*} \\
\sim 767^{*}\end{array}$ \\
\hline & Dow Corning & $\begin{array}{l}\text { Кислород } 2[9] \\
\text { Этан } \sim 11.8[9] \\
\text { Бутан } \sim 36[9] \\
\text { Толуол } \sim 964[9]\end{array}$ & $\begin{array}{l}\text { Кислород } 4 \\
\text { Этан -up to } 130 \\
\text { Бутан -up to } 1200 \\
\text { Толуол -up to } 1 \cdot 10^{5}\end{array}$ & $\sim 5 \cdot 10^{4 *}$ \\
\hline
\end{tabular}

Примечание. * - результаты, полученные в настоящей работе 


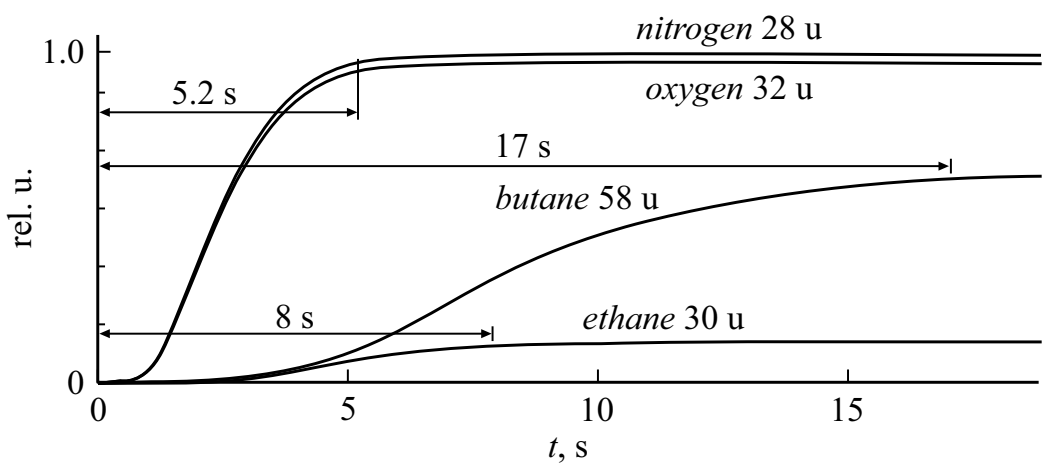

$a$

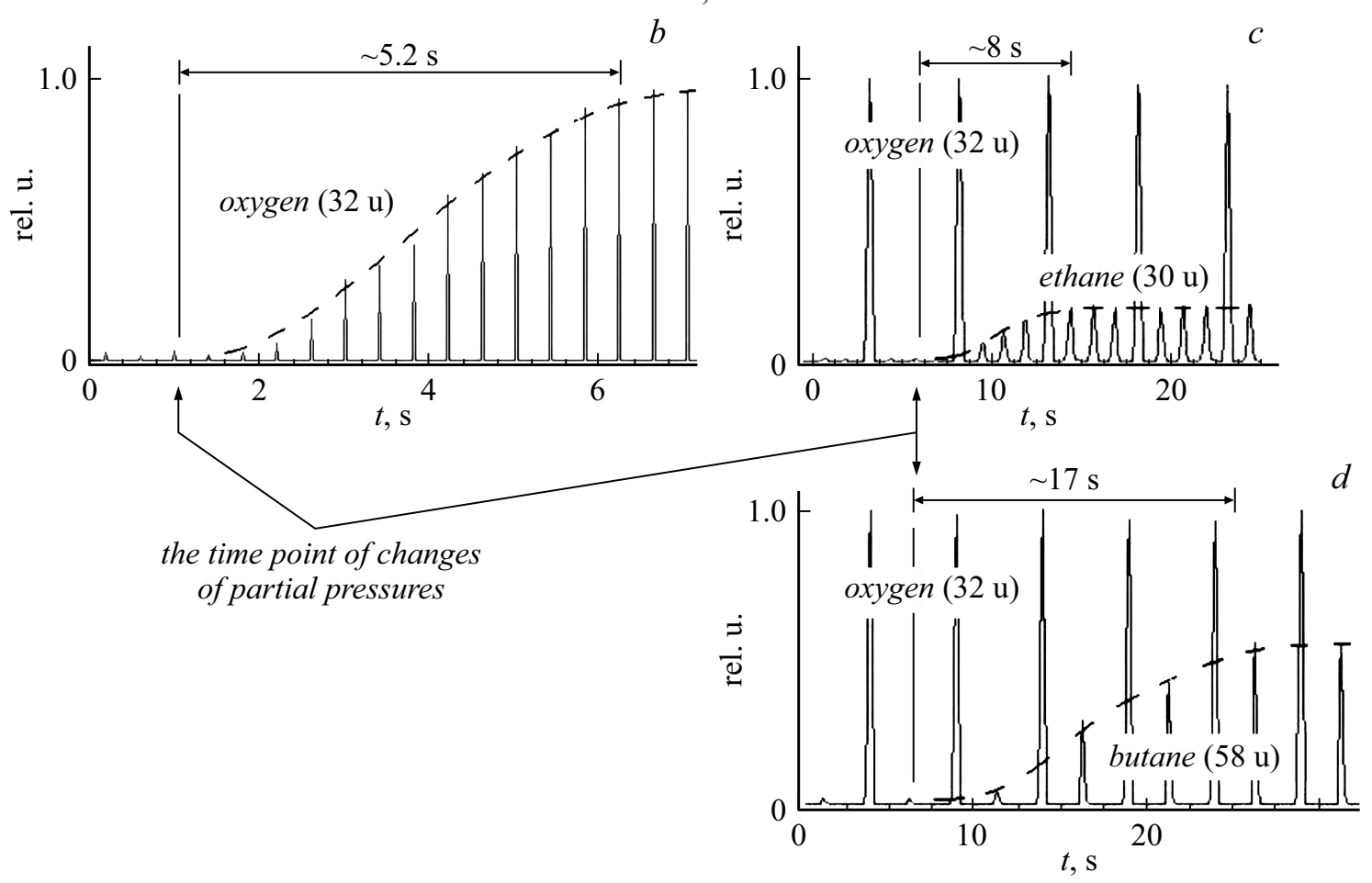

Рис. 4. Результаты численного моделирования отклика масс-спектрометра по характерным пикам азота (28u), кислорода (32 u), этана $(30 \mathrm{u})$ и бутана $(58 \mathrm{u})$ после ступенчатого изменения их концентрации в пробе соответственно от 0 до $78 \%$, от 0 до $21 \%$, от 0 до $0.33 \%$ и от 0 до $0.33 \%(a)$. Экспериментальные данные отклика масс-спектрометра по характерным пикам кислорода $(b)$, этана $(c)$ и бутана $(d)$, при тех же изменениях в пробе. Использована мембрана SSP-M100 толщиной $100 \mu \mathrm{m}$.

\section{Экспериментальная проверка результатов расчета}

Параметры первой мембраны с учетом эквивалентной схемы (рис. 2) и особенностей ее конструкции выбираются близкими к параметрам второй мембраны, а величина объема между ними - возможно меньшей, чтобы уменьшить задержку отклика системы ввода. Величина проводимости канала откачки из оъема между мембранами выбирается такой, чтобы поддерживать в нем давление $P_{V}$. Выбор толщины мембраны $l$, с одной стороны, должен отвечать требованию минимального времени отклика системы ввода, а с другой - прочности тонкого материала мембраны. Этим требованиям удовлетворяют мембраны от $l_{30}=30 \mu \mathrm{m}$. Однако производители мембран в настоящее время не выпускают материала толщиной менее 50-60 $\mu \mathrm{m}$ из-за ограниченного спроса на них. Тем не менее технологии их изготовления существуют, и следует на них рассчитывать.

На рис. 3 представлены расчетные значения плотностей потоков целевых соединений на входе в массспектрометр и величин относительного обогащения пробы этими соединениями в зависимости от давления $P_{V}$. В качестве целевых соединений рассматриваются бутан и толуол. В интервале давлений $0<P_{V}<P_{\mathrm{atm}} \sigma_{a}^{m} / \sigma_{j}^{m}$ величина относительного обогащения пробы близка к максимальному значению. В области давлений $P_{V}>P_{\text {atm }} \sigma_{a}^{m} / \sigma_{j}^{m}$, с ростом $P_{V}$ величина потока $I_{j}$ приближается к постоянному значению

$$
I_{j} \delta I_{a} / \delta P_{V}^{*}\left(p_{j 0} / p_{a 0}\right)\left(\sigma_{j}^{m} / \sigma_{a}^{m}\right) P_{\mathrm{atm}},
$$

где $I_{a}-$ поток основного компонента пробы воздуха за второй мембраной, $p_{a 0}-$ парциальное давление 
основного компонента и $p_{j 0}-$ парциальное давление целевого соединения в исходной пробе соответственно.

Различие величин проницаемости материала мембраны для разных компонентов в пробе приводит к фракционированию состава перед первой мембраной и, как следствие, к снижению эффекта обогащения целевыми соединениями. Чтобы свести к минимуму это фракционирование для большей части исследуемых соединений, необходимо ограничить поток пробы со стороны минимального значения величиной:

$$
w_{\text {sampl }}>4 \cdot 10^{3} l_{30} l_{d} S q(1 \cdot \mathrm{bar} / \mathrm{min}),
$$

где $I_{a}^{\prime}$ - поток плотности основных компонентов воздушной смеси через первую мембрану, $l_{d}$ - толщина, $S q$ - площадь используемой мембраны.

В качестве тестовых соединений в эксперименте использовались такие соединения как этан, бутан и толуол. Этан и бутан имеют скромную величину проникающей способности через мембрану, и служат прежде всего для экспериментального подтверждения результатов численного моделирования. Толуол же обладает высокой проникающей способностью, как и значительная группа целевых соединений, рассматриваемых в медицине в качестве маркеров заболеваний. [15-17]. В табл. 1 представлена группа соединений (а точнее, характерных пиков их спектров масс), связанных с определенными заболеваниями, а также коэффициенты обогащения воздушной пробы этими соединениями относительно азота при прохождении через одну мембрану $\sigma_{j}^{m} / \sigma_{N}^{m}$. Из табл. 1 видно, что эффект обогащения воздушной пробы представленными целевыми соединениями составляет величину порядка той, которая определена для толуола.

Сопоставление результатов эксперимента и расчета проводилось для прототипа системы ввода с мембранами SSP-100 площадью $3 \mathrm{~cm}^{2}$, толщиной $100 \mu \mathrm{m}$, и мембранами DOW Corning площадью $6 \mathrm{~cm}^{2}$, толщиной $59 \mu \mathrm{m}$. В табл. 2 приведены значения проницаемостей мембран. Величина рабочей площади поверхности выбранных для испытания мембран объясняется вынужденным ограничением подводимой пробы, из-за большой величины проницаемости мембран для попадающих в масс-спектрометр паров воды и ее неопределенности, особенно при изучении выдыхаемого человеком влажного воздуха.

В эксперименте с мембраной SSP-100 в качестве тестовых соединений использовались соединения с небольшими значениями $\sigma_{j}^{m}$ (этан и бутан). Условия максимального относительного обогащения проб указанными соединениями уже при давлении $P_{v} \sim 4$ mbar удовлетворяют соотношению (1). На рис. 4 представлены результаты расчета и эксперимента. Для того чтобы соотнести результаты экспериментальных данных и результаты расчета, авторам было необходимо учесть все коэффициенты перехода от потоковой характеристики каждого определяемого соединения к интенсивности его

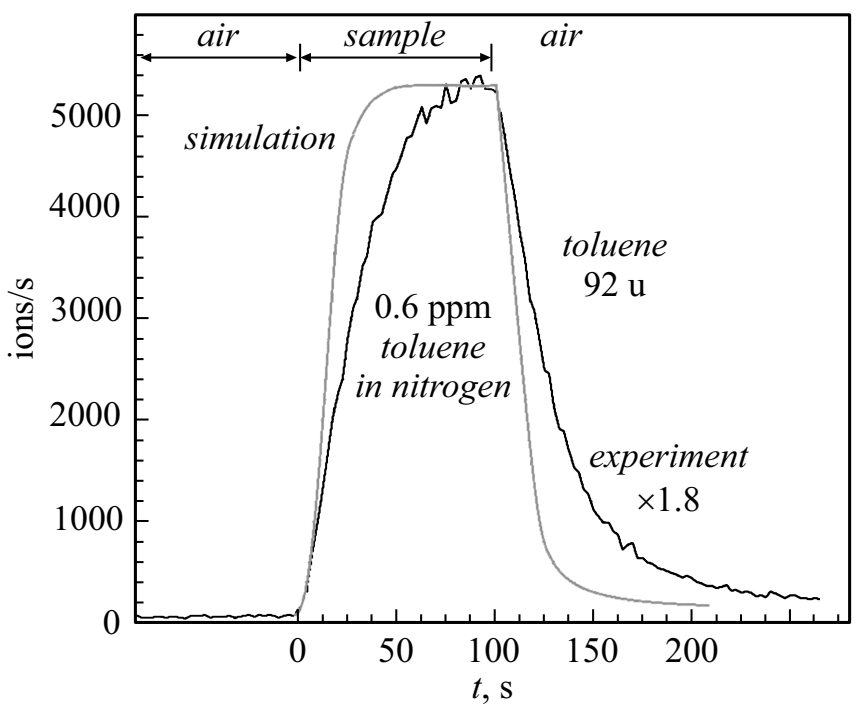

Рис. 5. Экспериментальные данные и результаты численного моделирования отклика масс-спектрометра по характерному пику толуола $(92 \mathrm{u})$ на изменение его концентрации от 0 до 0.6 ppm в атмосфере азота.

характерного пика в спектре масс. При этом учитывалось сечение ионизации соединения при выбранной энергии ионизации, вклад характерного пика в площадь его полного масс-спектра, а также особенности функции отклика используемого в исследовании массспектроиметра. Поскольку указанные параметры являются результатом исследований, проводимых авторами, то эти неопределенности складываются из погрешностей проведенных измерений.

Из табл. 2 видно, что величина относительного обогащения пробы целевыми соединениями достигает максимума (этан) или приближается к нему (бутан). Согласие между расчетом и экспериментом наблюдается не только по величине обогащения пробы в процессе подачи ее в масс-спектрометр, но и по величине запаздывания отклика системы относительно момента изменения состава пробы (рис. 4).

Эксперимент с применением мембраны DOW Corning демонстрирует ее возможность многократно обогащать пробу соединениями, для которых велика величина $\sigma_{j}^{m}$ (например, толуол), даже если условия напуска не обеспечивают максимального обогащения (1). Результаты эксперимента при давлении $P_{v} \sim 4 \cdot 10^{2} \mathrm{~Pa}$ и ограниченной площади мембраны представлены на рис. 5 и в табл. 2. В эксперименте с толуолом наблюдается отличие от расчетного значения, как в отношении обогащения пробы ( вдвое ниже расчетного), так и в отношении времени запаздывания отклика сигнала ( вдвое превышает расчетное). Такое отличие прежде всего связано с недостатком ротации газовой смеси перед мембранами, что особенно сказывается на фракционировании в межмембранном объеме соединений с высокими значениями $\sigma_{j}^{m}$. 

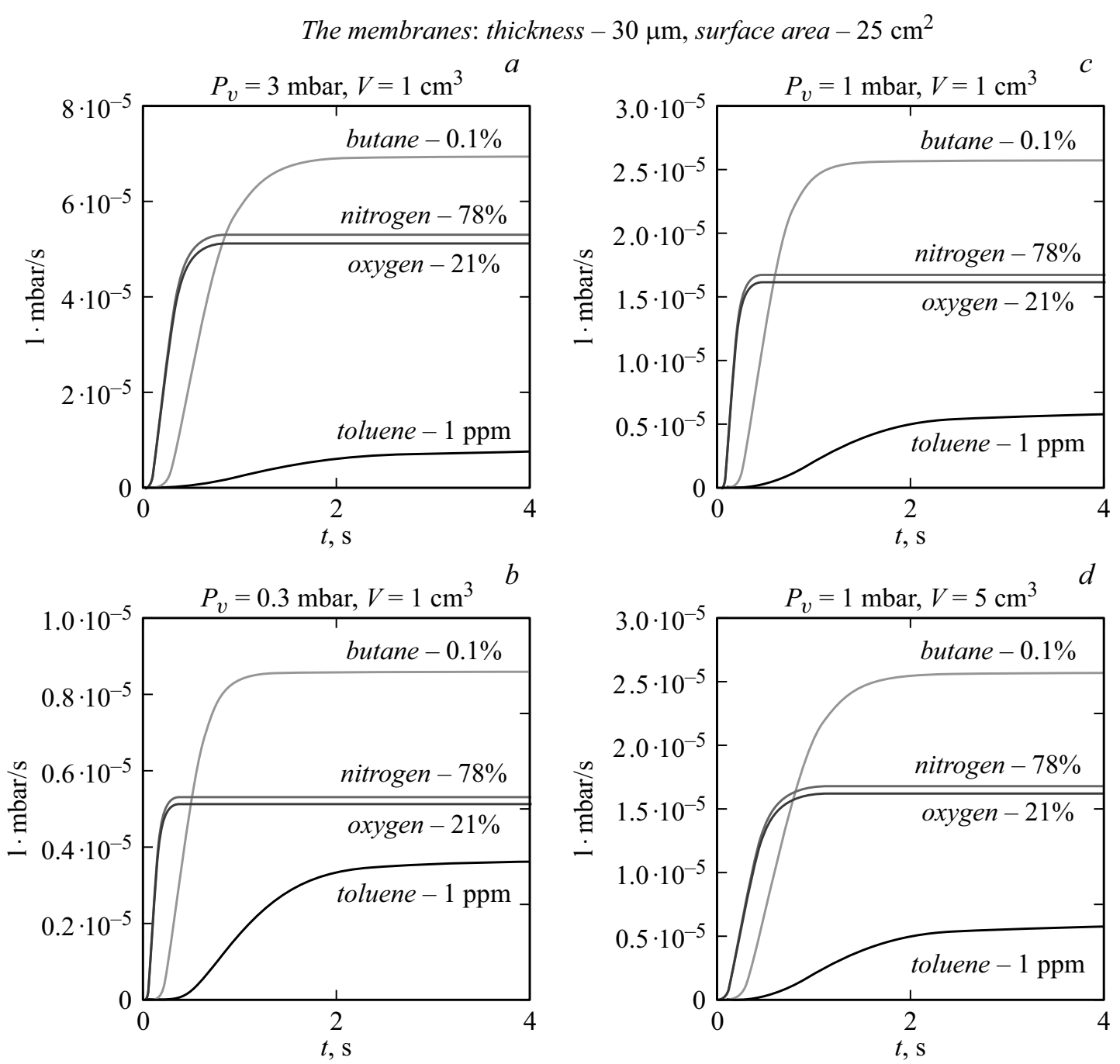

Рис. 6. Результаты численного моделирования системы ввода с оптимальными параметрами. Величиа отклика системы ввода пробы после скачкообразного изменения концентрации соединения в пробе при разных давлениях $P_{v}(a-c)$ и отличающихся мемжмембранных объемах $V(c, d)$.

\section{Выбор параметров системы ввода}

При определении следовых концентраций VOCs в пробах воздуха размеры мембран определяются допустимым потоком пробы в камеру масс-спектрометра. Большую часть потока воздушной пробы, проходящего в масс-спектрометр через двухмембранную систему ввода, составляют такие компоненты, как азот и пары воды. Это объясняется тем, что азот является преобладающим в исходной смеси и, несмотря на низкую проникающую способность через мембрану, остается таковым в потоке в вакуумную камеру масс-спектрометра. Существенную конкуренцию азоту составляют пары воды, абсолютная концентрация которых в атмосферном воздухе может колебаться от $10^{-4}$ до $10^{-3}$ (и даже $10^{-2}$ ) g/g, а проникающая способность через мембрану $\sigma_{\text {vaporm }}$ на два порядка выше, чем у азота. В связи с этим использование двухмембранной системы приводит к тому, что поток пара в масс-спектрометр может существенно превышать поток азота, а величина этого потока находится в зависимости от влажности исследуемого воздуха. Потоки именно этих двух составляющих воздушной смеси и необходимо соизмерять с производительностью высоковакуумного насоса, который предполагается использовать при применении двухмембранной системы.

Ограничим наше рассмотрение применением системы ввода для масс-спектрометров, в высоковакуумной части которых поддерживается рабочее давление $P_{m s} \leq 10^{-4} \mathrm{~Pa}$, а производительность высоковакуумных насосов по азоту $w$ составляет величину, способную поддерживать это давление при потоке азота в прибор на уровне $\sim 1.4 \cdot 10^{-3} l \mathrm{~Pa} / \mathrm{s}$. При этом выбор производительности насоса зависит от наибольшей предполагаемой влажности воздушных проб, на работу с которыми рассчитан инструмент. Такой выбор позволит учесть вклад паров воды, которые становятся преобладающими в потоке на входе в масс-спектрометр из-за значительной величины $\sigma_{\text {vaporm }} \sim 100 \sigma_{a}^{m}$. 
Известно, что для большей части целевых соединений проницаемость мембраны ограничена соотношением $20 \sigma_{a}^{m}<\sigma_{j}^{m}<1000 \sigma_{a}^{m}[9]$, а относительное обогащение воздушной пробы этими соединениями может приближаться к максимальному значению $\left(\sigma_{j}^{m} / \sigma_{a}^{m}\right)^{2}$ только в случае выполнения соотношения (1). Поэтому необходимым условием приблизиться к максимальной относительной чувствительности для целевых соединений, включая те, для которых отношение $\sigma_{j}^{m} / \sigma_{a}^{m}$ близко к максимальному, является поддержание величины давления $P_{V} \leq 1 \cdot 10^{2} \mathrm{~Pa}$. При выборе мембран остановимся на материале DOW Corning, толщиной $l_{30}=30 \mu \mathrm{m}$, который следует и допустимо использовать для достижения максимального эффекта обогащения большей части летучих соединений, а также для обеспечения экспрессности измерений при приемлемой механической прочности интерфейса. Площадь мембраны $S q$ с учетом результатов работы [9] составляет $\sim 25 \mathrm{~cm}^{2}$. На рис. 6 можно наблюдать результаты расчета отклика системы ввода, проведенного по предложенному в настоящей работе алгоритму. Показана зависимость характеристик системы ввода пробы от давления $P_{V}$ и объема $V$ при допустимой для выбранных условий эксперимента площади мембранного интерфейса.

\section{Заключение}

В работе рассмотрен один из путей снижения пределов определения летучих органических соединений в воздушных пробах масс-спектрометрическим способом за счет применения системы ввода, включающей две мембраны, при минимально допустимой величине задержки отклика инструмента. Параметры системы могут быть оценены с приемлемой точностью благодаря представленному в работе алгоритму расчета. Расчет по предложенному алгоритму позволяет оценить степень вляния каждого из параметров и выбрать режим максимально возможного потока целевого соединения в массспектрометр, а также минимальную задержку отклика на изменение состава исходной пробы. Наибольшее систематическое отклонение характеристик реального потока от данных расчета наблюдается для соединений с максимальными значениями $\sigma_{j}^{m}$ и в предложенной конструкции находится в пределах 100\%. Такое отличие можно рассматривать как приемлемое, если учесть, что в процессе прохождения через интерфейс проба обогащается указанными соединениями до ста тысяч раз.

Одним из перспективных приложений обсуждаемой системы ввода является ее применение для обнаружения в пробах воздуха летучих органических соединений с высокими значениями $\sigma_{j}^{m}>100 \sigma_{a}^{m}$ на уровне долей ppb. К подобным соединениям прежде всего следует отнести большую группу биомаркеров заболеваний, содержащихся в выдыхаемом человеком воздухе. Важным аргументом в пользу применения рассматриваемой системы для диагностики патологий по выдыхаемому воздуху является и то, что она допускает проведение анализа при значительном потоке подводимой пробы ( $1 \mathrm{l} / \mathrm{min})$, что необходимо для обеспечения низких пределов обнаружения. Сравнительная простота инструмента позволяет рассчитывать на возможность его широкого применения.

Авторы благодарят фирму DOW CORNING за содействие в выборе материала мембраны и предоставлении его для настоящего проекта, а также Фонд содействия развитию малого бизнеса за частичную финансовую поддержку контрактом ВН 0021774.

\section{Список литературы}

[1] Lindinger W., Hansel A., Jordan A. // Int. J. Mass Spectrom. Ion Processes. 1998. Vol. 173. P. 191-241.

[2] Adams N.G., Smith D. // Int. Ion Phys. 1976. Vol 21. P. 349-359.

[3] Lourenço C., Turner C. // Metabolites. 2014. Vol. 4. P. 465-498.

[4] Amann A., Smith D. Volatile biomarkers (Chapters 3,4). Elsiver, 2013. $565 \mathrm{p}$.

[5] Boots A.W., Berkel J.J.B.N., Dallinga J.W., Smolinska A., Wouters E.F., Schooten F.J. // J. Breath Res. 2012. Vol. 6. P. 027108.

[6] 6th International Conference on Proton Transfer Reacrevoltqnton Mass Spectrometry and its Applications. Editors: Hanse A., Dunk J. Oinnsbruck university press, Obergurgl, Austria, 2013. 248 p.

[7] Johnson R.C., Cooks R.G., Allen T.M., Cisper M.E., Hemberger P.H. // Mass Spectrometry Reviews. 2000. Vol. 19. N 1. P. 1-37.

[8] Overney F.L., Enke C.G. // J. Am. Soc. Mass Spectrom. 1996. Vol. 7. P. 93-100.

[9] LaPack M.A., Tou J.C., Mc Guffin V.L., Enke C.G. // J. Membr. Sci. 1994. Vol. 86. P. 263-280.

[10] Ketola R.A., Kotiaho T., Cisper M.E., Allen T.M. // J. Mass Spectrom. 2002. Vol. 37. P. 457-476.

[11] Viktorova O.S., Kogan V.T., Manninen S.A., Kotiaho T., Ketola R.A., Dubenskii B.M., Parinov S.P., Smirnov O.V. // J. Am. Soc. Mass Spectrom. 2004. Vol. 15. P. 823-831.

[12] Коган В.Т., Викторова О.С., Викторов И.В. // ПТЭ. 2005. Вып. 1. С. 1001-1004.

[13] Llewellyn P.M., Littlejohn D.P. Abstracts of Papers. Conf. on Analytical Chemistry and Applied Spectroscopy, Pittsburgh, USA. 1966.

[14] Коган В.Т., Козленок А.В., Чичагов Ю.В., Антонов А.С., Лебедев Д.С., Богданов А.А., Морошкин В.С., Березина А.В., Викторова-Леклерк О.С., Власов С.А., Тубольцев Ю.В. // ЖТФ. 2015. Т. 85. Вып. 10. С. 135-140.

[15] Buszewski B., Kesy M., Ligor T., Amann A. // Biomed. Chromatogr. 2007. Vol. 21. P. 553.

[16] Boots A.W., van Berkel J.J.B.N., Dallinga J.W., Smolinska A., Wouters E.F., van Schooten F.J. // J. Breath Res. 2012. Vol. 6. P. 027108.

[17] Miekisch W., Schubert J.K., Noeldge-Schomburg G.F. // Clin. Chim. Acta. 2004. Vol. 347. P. 25-39.

[18] Specialty Silicone Products, Inc. http://www.sspinc.com/

[19] Коган В.Т., Антонов А.С., Лебедев Д.С., Козленок А.В., Власов С.А., Чичагов Ю.В., Викторов И.В. // ПТЭ. 2013. Вып. 4. С. 112-116. 